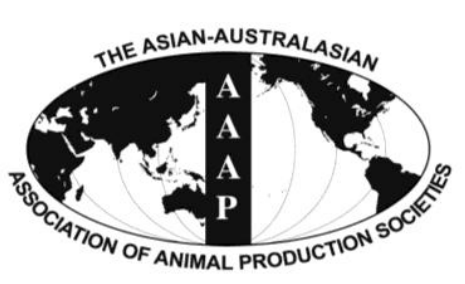

Open Access

Asian Australas. J. Anim. Sci.

Vol. 27 , No. $7:$ : 1057-1068 July 2014

http://dx.doi.org/10.5713/ajas.2013.13702

www.ajas.info

pISSN 1011-2367 elSSN 1976-5517

\title{
Nutritional Factors Affecting Abdominal Fat Deposition in Poultry: A Review
}

\author{
A. M. Fouad* and H. K. El-Senousey \\ Department of Animal Production, Faculty of Agriculture, Cairo University, Giza 12613, Egypt
}

\begin{abstract}
The major goals of the poultry industry are to increase the carcass yield and to reduce carcass fatness, mainly the abdominal fat pad. The increase in poultry meat consumption has guided the selection process toward fast-growing broilers with a reduced feed conversion ratio. Intensive selection has led to great improvements in economic traits such as body weight gain, feed efficiency, and breast yield to meet the demands of consumers, but modern commercial chickens exhibit excessive fat accumulation in the abdomen area. However, dietary composition and feeding strategies may offer practical and efficient solutions for reducing body fat deposition in modern poultry strains. Thus, the regulation of lipid metabolism to reduce the abdominal fat content based on dietary composition and feeding strategy, as well as elucidating their effects on the key enzymes associated with lipid metabolism, could facilitate the production of lean meat and help to understand the fat-lowering effects of diet and different feeding strategies. (Key Words: Abdominal Fat Content, Lipogenesis, Lipolysis, Nutritional Factors, Poultry)
\end{abstract}

\section{INTRODUCTION}

In 1953, chicken producers required more than 70 days to rear chickens to a final body weight of $1.5 \mathrm{~kg}$. By 2001 , however, chicken producers could rear chickens to their final body weight of $2.5 \mathrm{~kg}$ in a fattening period of only 42 days (Havenstein et al., 2003; Flock et al., 2005). Chickens have been genetically improved for increased body weight gain, feed efficiency, growth rate, and breast muscle weight to meet the requirements of consumers (Wang et al., 2012). These selection processes have produced modern commercial chicken lines with a higher growth rate, breast meat yield and better feed conversion rates, and a higher body fat compared with unselected lines (Baéza and Le Bihan-Duval, 2013). However, the excessive fat in modern poultry strains has been one of the major problems facing the poultry industry (Zhou et al., 2006). For example, Choct et al. (2000) found that modern broiler strains contain $15 \%$ to $20 \%$ fat and $>85 \%$ of this fat is not physiologically required for body function. In general, excessive fat deposition is an unfavorable trait for producers and

* Corresponding Author: A. M. Fouad. Tel: +20-2-35440696, Fax:+20-2-35717355, E-mail: ahmedmfouad2000@yahoo.com Submitted Nov. 5, 2013; Revised Jan. 20, 2014; Accepted Feb. 19, 2014 consumers because it is considered to be wasted dietary energy and a waste product with low economic value, which also reduces the carcass yield and affects consumer acceptance (Emmerson, 1997). Laying hens also exhibit excessive fat accumulation, which negatively affects their reproductive performance (Xing et al., 2009).

In avian species, most fatty acids are synthesized in the liver and transported via low-density lipoproteins or chylomicrons for storage in adipose tissues as triglycerides (Hermier, 1997). The abdominal fat tissue is crucial in poultry because it grows faster compared with other fat tissues (Butterwith, 1989). The abdominal fat pad is a reliable parameter for judging total body fat content because it is linked directly to total body fat content in avian species (Becker et al., 1979; Thomas et al., 1983).

Nutritional factors can regulate body fat deposition. In general, it is accepted that inhibiting the absorption of dietary fat and fatty acid synthesis, and/or promoting fatty acid $\beta$-oxidation reduces abdominal fat deposition by decreasing the size and/or number of abdominal adipose cells (Figure 1). Thus, this review discusses the nutritional factors that affect the abdominal fat content and how these factors can regulate abdominal fat deposition in poultry in a beneficial manner. 


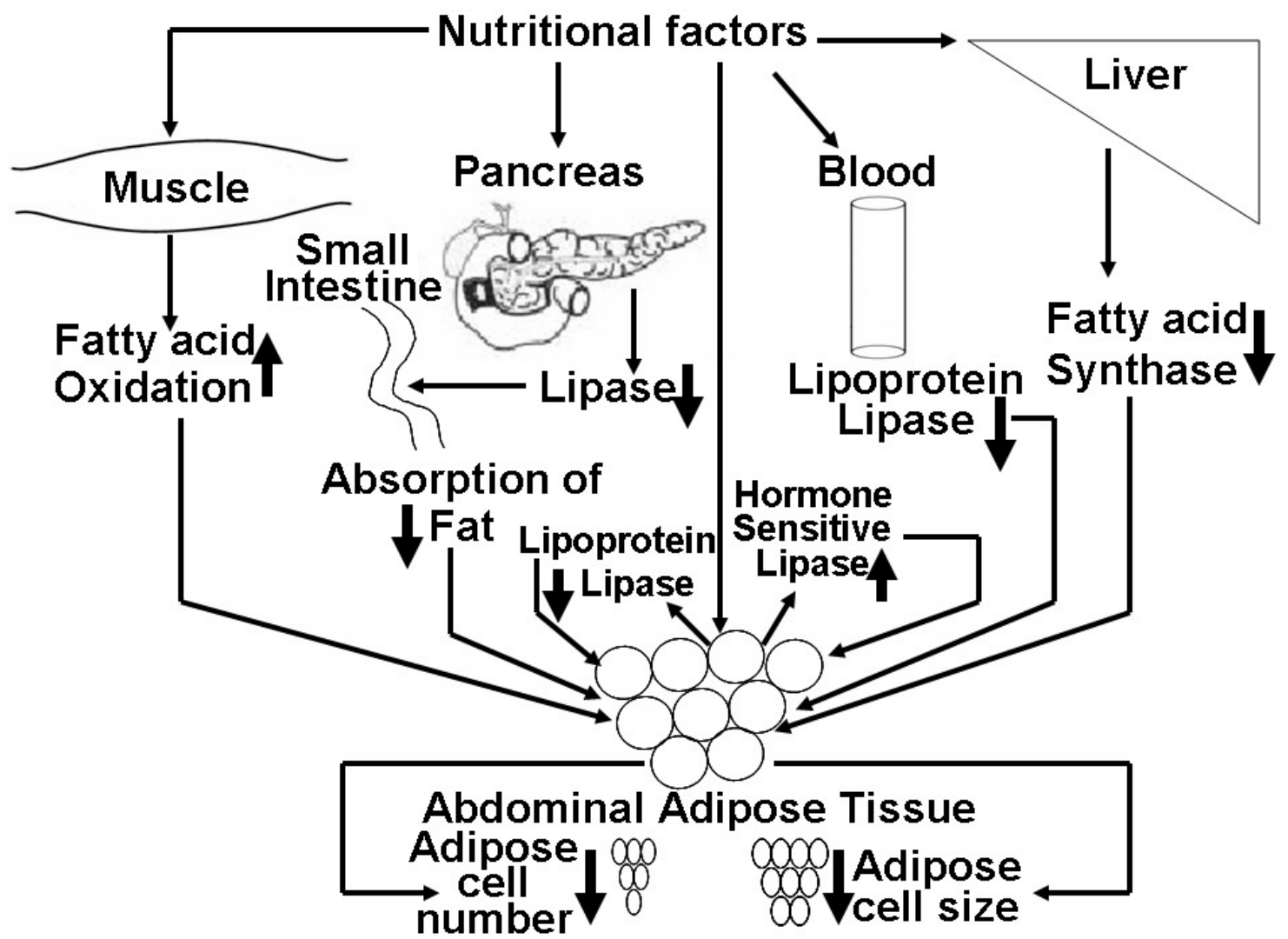

Figure 1. The mechanisms of nutritional factors that beneficially regulate abdominal fat deposition. Nutritional factors may: reduce fatty acid synthesis in the liver (the crucial site for fatty acid synthesis); suppress the secretion of pancreatic lipase, which reduces fat absorption; increase fatty acid $\beta$-oxidation in the muscles; inhibit the activity of lipoprotein lipase in the blood or abdominal adipose tissue; and/or enhance the activity of hormone-sensitive lipase in abdominal adipose tissue, which finally leads to a reduction in abdominal adipose tissue by decreasing the size and/or number of abdominal adipose cells. $\uparrow$, enhanced; $\downarrow$, inhibited.

\section{DISCUSION}

\section{Nutrient levels on abdominal fat}

Energy: In avian species, dietary energy level can be used to reduce the feed cost per unit of poultry product through its effects on feed intake and feed conversion, so it is one of the most important factors that can be modified to reduce body fat deposition. Kassim and Suwanpradit (1996) showed that reducing the energy level from 3,200 to 3,000 $\mathrm{kcal} / \mathrm{kg}$ in broiler chickens from 21 to 42 days of age significantly reduced the abdominal fat percentage and total body fat deposition without any negative effects on the average daily gain, feed intake, or dressing percentage. Rabie and Szilagyi (1998) also found that the abdominal fat deposition was reduced significantly by decreasing dietary energy level from 3,227 to $3,059 \mathrm{kcal} / \mathrm{kg}$ in broilers from 18 to 53 days of age had no significant impact on the final live weight, carcass yield, or breast meat. Similar results were reported by Fan et al. (2008) who found that the breast and leg muscle percentages did not change whereas the abdominal fat weight relative to the live body weight was reduced significantly by decreasing dietary energy level from 2,900 to $2,700 \mathrm{kcal} / \mathrm{kg}$ in ducks from 14 to 42 days of age. Furthermore, Xie et al. (2010) showed that feeding ducklings from hatching to 21 day of age with a diet containing $2,747 \mathrm{kcal} / \mathrm{kg}$ led to a significant reduction in the abdominal fat content (percentage of live weight) without any significant reduction in the breast or leg meat compared with a diet containing 3,045 kcal $/ \mathrm{kg}$. Reducing dietary energy level leads to a reduction in total body fat deposition by decreasing the activity levels of a number of enzymes linked to hepatic lipogenesis, including nicotinamide adenine dinucleotide phosphate- malate dehydrogenase, glucose-6-phosphate dehydrogenase (G-6-PDH), 6phosphogluconate dehydrogenase, and fatty acid synthase (FAS) in chickens (Tanaka et al., 1983). The FAS is a crucial enzyme in the de novo lipogenesis pathway in the liver of chickens. The ability of chickens to synthesize fatty acid deposits in the body is determined by the activity of FAS in the liver (Back et al., 1986). Therefore, the formulation of poultry diets to meet their energy requirements based on guidelines for specific strains is recommended to reduce body fat deposition, while increasing dietary energy content to improve feed 
conversion leads to the increased deposition of fat.

Protein: Protein is the most expensive component of poultry diets. Increasing dietary protein content improves the average daily gain, carcass yield, and carcass quality by increasing protein content while reducing body fat deposition. Kassim and Suwanpradit (1996) found that reducing dietary protein level from $23 \%$ to $20 \%$ crude protein $(\mathrm{CP})$ during the starter phase, and from $20 \%$ to $18 \%$ $\mathrm{CP}$ during the finisher phase, led to a significant increase in the abdominal fat content. In a comparison of low-protein and normal-protein diets in broiler chickens, Collin et al. (2003) found that low-protein diets caused a significant increase in the abdominal fat content percentage. Yalçin et al. (2010) also found that feeding broiler chickens diets containing $19.2 \%, 16.6 \%$, and $15.5 \% \mathrm{CP}$ (low protein) led to an increase in total carcass fat deposition compared with chickens fed diets containing $22.9 \%, 19.9 \%$, and $18.2 \% \mathrm{CP}$ (the standard recommended by NRC [1994]) in the starter, grower, and finisher phases, respectively. Increasing dietary protein level in the diets of broiler chickens to $26.6 \%$, $23.5 \%$, and $20.7 \%$ in the starter, grower, and finisher phases led to a reduction in total carcass fat deposition compared with diets formulated according to NRC (1994) (Yalçin et al., 2010). Moreover, Jlali et al. (2012) found that increasing dietary CP level from $17 \%$ to $23 \%$ in fat and lean broiler chickens from 21 to 63 days of age caused a significant reduction in abdominal fat deposition. Therefore, dietary protein content must play a direct or indirect role in the regulation of lipid metabolism. However, Adams and Davis (2001) and Rosebrough et al. (2002) found that reducing dietary $\mathrm{CP}$ protein content upregulated malic enzyme mRNA expression and elevated the activity of malic enzyme in the liver of broilers compared with the control, whereas increasing dietary $\mathrm{CP}$ protein content downregulated malic enzyme mRNA expression and decreased the activity of malic enzyme in the liver of broilers compared with the controls. Choi et al. (2006) also showed that increasing dietary protein content caused a significant reduction in FAS mRNA expression in the livers of broiler chickens compared with the controls. Furthermore, Rosebrough et al. $(2008 ; 2011)$ found that feeding broilers a diet containing a high $\mathrm{CP}$ level suppressed the mRNA expression of hepatic malic enzyme, acetyl coenzyme carboxylase (ACC), and FAS in a comparison of lowprotein and high-protein diets. Therefore, dietary protein level affected body fat deposition directly. Thus, it is better to meet the protein requirements of birds to produce high quality meat with low fat deposition.

Amino acids: Methionine, the first-limiting amino acid in poultry diets, is important because of its effects on growth performance and also due to its effectiveness for producing lean meat (Takahashi et al., 1995). However, Corzo et al. (2006), Yao et al. (2006), and Zhan et al. (2006) reported significant increases in the abdominal fat percentage after feeding chickens methionine-deficient diets. By contrast, Xie et al. (2006) and Wang et al. (2010) found that increasing the methionine concentration in the diet caused a significant linear decrease in the abdominal fat content in their studies, which estimated the methionine requirements for White Pekin ducks from 21 to 48 days of age and for Yangzhou geese from 28 to 70 days of age. Andi (2012) reported that dietary L-methionine supplementation in broiler chickens led to a significant decline in body fat content. Changes in lipogenesis and/or lipolysis may be associated with the fat-lowering effect of dietary L-methionine. Takahashi and Akiba (1995) found that dietary L-methionine supplementation regulated the body fat content by reducing the activity of FAS (lipogenesis) and increasing the activity of hormonesensitive lipase (HSL) (lipolysis).

In addition to its role as a limiting amino acid in poultry diets because of its effects on growth performance, lysine has a prominent role in meat quality by enhancing muscle $\mathrm{pH}$, increasing protein deposition, and reducing the waterholding capacity (Berri et al., 2008; Tesseraud et al., 2009). The inclusion of lysine in poultry diets significantly enhances lean meat production. In 1990, Moran and Bilgili demonstrated a significant reduction in the carcass fatness by adding L-lysine. Grisoni et al. (1991) showed that increasing the lysine concentration in chicken diets reduced abdominal fat deposition. Attia (2003) found a significant increase in the abdominal fat percentage in meat-type ducks fed a lysine-deficient diet while the addition of lysine eliminated this effect. Moreover, Berri et al. (2008) and Nasr and Kheiri (2011) showed that dietary L-lysine supplementation lowered fat deposition in broiler chickens. Therefore, lysine can be added to poultry diets to promote lean meat production by reducing carcass fatness via lipogenesis inhibition (Grisoni et al., 1991).

Arginine is an essential amino acid that has multiple functions in poultry production, which has been implicated in reduced carcass fat deposition (Fouad et al., 2012). Corzo et al. (2003) indicated that the inclusion of arginine in broiler chicken diets at higher levels than NRC (1994) recommendations could reduce carcass fatness. In Japanese quails, Al-Daraji et al. (2011) found a significant reduction in the abdominal fat content as a percentage of body weight at 42 day of age after injections of $2.0 \%$ arginine at 0 days of incubation. In meat-type ducks, Wu et al. (2011) reported that providing $1.0 \%$ additional arginine above the NRC (1994) recommendations reduced the abdominal fat content and carcass fatness by decreasing the activities of malate dehydrogenase (MDH), G-6-PDH, and FAS (lipogenesis). Fouad et al. (2013) recently reported that dietary arginine inclusion at $0.25 \%$ reduced abdominal fat deposition by suppressing hepatic FAS mRNA expression and enhancing 
carnitine palmitoyl transferase I (CPT1) and L-3- the activity of HSL in the abdominal fat. hydroxyacyl-CoA dehydrogenase (3HADH) mRNA expression in the hearts of broiler chickens. CPT I and L3HOAD are recognized as the main enzymes involved with $\beta$-oxidation (Eaton, 2002). In avian species, therefore, dietary L-arginine supplementation inhibits hepatic FAS mRNA expression and improves CPT1 and 3HADH mRNA expression, which causes a reduction in the abdominal fat content by reducing the size of abdominal adipose cells ( $\mathrm{Wu}$ et al., 2011; Fouad et al., 2013). At present, only three amino acids (methionine, lysine, and arginine) are known to beneficially regulate body fat deposition in poultry. Therefore, the inclusion of the optimal levels of these three amino acids in poultry diets should be ensured to avoid problems linked to fatness.

Minerals: Manganese (Mn) is a necessary trace mineral in poultry nutrition because of its central role in carbohydrate and lipid metabolism (Klimis-Tavantzis et al., 1983). $\mathrm{Mn}$ is also a powerful antioxidant that can be added to chicken diets to improve the meat shelf-life (Li et al., 2011). Moreover, $\mathrm{Mn}$ is effective for reducing the body fat content in broiler chickens. Sands and Smith (1999) first observed that adding $\mathrm{Mn}$ in the form of $\mathrm{Mn}$ proteinate at $240 \mathrm{mg} / \mathrm{kg}$ diet reduced the abdominal fat content as a percentage of the carcass weight. Lu et al. (2006) designed an experiment that used chickens to investigate the effects of different levels of inorganic Mn (0, 100, 200, 300, 400, and $500 \mathrm{mg} / \mathrm{kg}$ ) on the abdominal fat content and to understand the effects of Mn on lipid metabolism. They reported that addition of $\mathrm{Mn}$ at $100 \mathrm{mg} / \mathrm{kg}$ to broiler diets that contained $23 \mathrm{mg} \mathrm{Mn} / \mathrm{kg}$ significantly reduced the carcass fatness by downregulating the activity of lipoprotein lipase (LPL) in the abdominal fat. In avian species, it is well documented that fatty acids synthesized in the liver or those derived from the diet are transported to the adipose tissues via low-density lipoproteins or chylomicrons before storage in the adipose tissue, although only free fatty acids can pass through the adipocyte membranes so LPL hydrolyzes the triglycerides of chylomicrons and low-density lipoproteins to produce free fatty acids and glycerols, which are esterified into triglycerides in adipose tissues (Hermier, 1997). Therefore, LPL is necessary for the uptake of fatty acids by adipose tissues. It is well known that the bioavailability of organic minerals is higher compared with inorganic minerals. Thus, Lu et al. (2007) conducted an experiment to compare the effect of Mn sulphate (inorganic $\mathrm{Mn}$ ) and $\mathrm{Mn}$ amino acid (organic $\mathrm{Mn}$ ) on abdominal fat deposition. They found that the Mn source (organic or inorganic) did not affect fat deposition, whereas the concentration of $\mathrm{Mn}$ in the diet had a significant effect on the body fat content, i.e., diets supplemented with $100 \mathrm{mg}$ Mn from organic or inorganic sources reduced body fat deposition by decreasing the activity of LPL and elevating

\section{Fat types on abdominal fat}

Fats and oils are used in poultry diet formulations to enhance the palatability of the diet, the absorption of fatsoluble vitamins, and to regulate the passage rate of the digesta in the gastrointestinal tract. They are also the most concentrated sources of energy (Baião and Lara, 2005). In avian species, the amount of fat that accumulates in the body depends on the available plasma lipid substrate, which originates from the diet or de novo lipogenesis in the liver (Hermier, 1997). Therefore, the sources of lipids in poultry diets may affect their total body fat deposition. Sanz et al. (1999; 2000a) observed that a diet containing tallow or lard (saturated fat) achieved the same growth performance as a diet that contained sunflower oil (unsaturated fat), whereas the abdominal fat content was significantly lower in the chickens fed diets containing sunflower oil. Experimental evidence indicated that dietary inclusion of sunflower oil was effective for reducing total body fat deposition compared with a diet containing tallow, but this raised the question of why the inclusion of unsaturated fats in the diets of broiler chickens led to a lower accumulation of body fat compared with the inclusion of saturated fats. However, Sanz et al. (2000b) found that the inclusion of sunflower oil in the diet of broilers led to a significant reduction in the abdominal fat percentage by inhibiting the activity FAS in the liver and enhancing the activities of CPT I and L3HOAD in the heart compared with the inclusion of tallow in the diet. Therefore, unsaturated fats reduce abdominal fat deposition and total body fat via this mechanism, in contrast to saturated fats. However, the next question was whether all unsaturated fats reduce abdominal fat deposition in broiler chickens. Therefore, Crespo and Esteve-Garcia (2001; 2002a) fed chickens diets supplemented with tallow (a rich source of saturated fatty acids), olive oil (a rich source of monounsaturated fatty acids), sunflower oil (a rich source of n-6 type polyunsaturated fatty acids), and linseed oil (a rich source of n-3 type polyunsaturated fatty acids). They reported that chickens fed diets supplemented with sunflower or linseed oils had a significant reduced in abdominal fat percentage and other fat depots, including mesenteric and neck fat, compared with chickens fed diets supplemented with tallow or olive oil. According to the results of Sanz et al. (2000b), the inclusion of sunflower oil in the diets of broilers promoted fatty acid oxidation and depressed fatty acid synthesis, so the abdominal fat percentage was decreased significantly. Linseed oil is a source of n-3 type polyunsaturated fatty acids while sunflower oil contains n-6 type polyunsaturated fatty acids. Thus, linseed oil could have reduced total body fat via the same mechanism as sunflower oil or other mechanisms. However, Crespo and 
Esteve-Garcia (2002b) found that inclusion of linseed oil in the diets of broilers led to a significant reduction in the abdominal fat percentage and a significant increase in fatty acid synthesis compared with tallow or sunflower oil, so linseed oil may reduce the abdominal fat percentage by elevating fatty acid $\beta$-oxidation. A similar result was reported by Newman et al. (2002) who found that, compared with the addition of tallow, the addition of sunflower or fish oil to the diets of broilers from 21 to 56 days of age reduced abdominal fat deposition, increased the consumption of oxygen, and decreased the rate of oxygen consumption relative to the rate of carbon dioxide production, which showed that polyunsaturated fatty acids activate fatty acid $\beta$-oxidation. Furthermore, Ferrini et al. (2010) reported that, compared with those fed diets containing tallow, the activity of L3HOAD increased significantly whereas the activities of MDH and G-6-PDH (lipogenic enzymes) did not change in chickens fed diets containing linseed oil. This showed that linseed oil reduces abdominal fat deposition by promoting fatty acid $\beta$ oxidation, rather than suppressing fatty acid biosynthesis. Therefore, polyunsaturated fatty acids (n-3 and n-6 types) that have many benefits for human health (Simopoulos, 2000) can be used during poultry production to rear poultry products containing the optimal ratio of polyunsaturated fatty acids (n-3 and n-6) but also to reduce fat deposition in the abdomen area of poultry, which will enhance the producer returns and consumer health.

\section{Conjugated linoleic acid on abdominal fat}

The source of oils is not the only factor that can affect abdominal fat deposition in broilers. The structure of oil (fatty acid isomers) is one of the most important factors that can affect body fat deposition in broilers, as demonstrated by Simon et al. (2000). Their results indicated that the inclusion of conjugated linoleic acid (CLA) in chicken diets reduced the skin fat (one of the most important fat depots in poultry) compared with diets that lacked linoleic acid. CLA is a common name for the positional and geometric isomers of linoleic acid (9cis, 12cis octadecadienoic acid; 18:2n 6). A similar result was also reported by Badinga et al. (2003) who found that CLA depressed fat deposition in the livers of chickens in a comparison of CLA and corn oil (a rich source of linoleic acid). Moreover, Szymczyk et al. (2001) showed clearly that the inclusion of $1.5 \%$ CLA in broiler diets significantly reduced the abdominal fat content compared with the control diet (0\% CLA). CLA can reduce abdominal fat deposition and lower total carcass fat deposition, as reported by Du and Ahn (2002). Zhang et al. (2009a) also supplemented 2.5\% CLA in the diet of geese, which resulted in a significant reduction in the abdominal fat content compared with supplementation with the same amount of soybean oil, which is a rich source of linoleic acid. Zhang et al. (2007) and Zhou (2008) showed that CLA reduced body fat deposition in unimproved chicken strains (local strains), which have a low capacity for synthesizing lipids compared with improved strains (modern commercial chickens), as demonstrated by Cui et al. (2012). Zhang et al. (2007) demonstrated that a Beijingyou chicken diet containing $1.0 \%$ CLA led to a significant reduction in the abdominal fat content compared with a control diet that contained corn oil. The abdominal fat deposition was reduced significantly by adding $3.0 \%$ CLA to the diets of Yellow-feather broiler chickens compared with control diets containing $3.0 \%$ rapeseed oil as a rich source of linoleic acid (Zhou, 2008). According to these studies, CLA is a new tool that can be used to reduce fatness in modern broiler chickens, although the question remains why the inclusion of CLA in broiler diets caused a significant reduction in the abdominal fat content and total body fat deposition. Zhang et al. (2007) suggested that CLA reduces total body fat deposition in broilers by suppressing the activity of LPL in the plasma, while Zhou (2008) suggested that CLA reduced abdominal fat deposition in Yellowfeather broiler chickens (local Chinese strain) by downregulating peroxisome proliferator-activated receptor $\gamma$ (PPAR $\gamma$ ) mRNA expression in abdominal adipose tissue. Royan et al. (2011) confirmed the findings of Zhou (2008) by testing the inclusion of CLA in the diet of a commercial strain of broiler chickens (Ross 308), which caused a significant reduction in the abdominal fat percentage by reducing PPAR $\gamma$ mRNA expression in the abdominal adipose tissue. In avian species, $\operatorname{PPAR} \gamma$, which regulates lipid metabolism and adipocyte differentiation, is strongly associated with abdominal fat deposition (Sato et al., 2009; Wang et al., 2008; Xiong et al., 2010). In geese, Zhang et al. (2009a) suggested that CLA reduces the abdominal fat pad percentage by activating fatty acid oxidation via an increase in the activity of CPT I. In avian species, we can conclude that CLA reduces total body fat deposition directly by reducing the activity of LPL and downregulating PPAR $\gamma$ mRNA expression, and indirectly by elevating the activity of CPT I.

\section{Various feed additives on abdominal fat}

Betaine (glycine betaine or trimethylglycine) is highly concentrated in sugar beet and is classified as a byproduct of sugar production. It is considered to be an important source for methyl donors in farm animals (Virtanen, 1995). In White Pekin ducks, Wang et al. (2004) detected a reduction in the abdominal fat yield percentage after the inclusion of betaine in their diet. Zhan et al. (2006) also found that adding betaine to a methionine-deficient diet significantly reduced the fat deposition in broiler chickens. $\mathrm{Su}$ et al. (2009) reported that feeding Landes geese a diet supplemented with betaine caused a significant decrease in 
the abdominal fat compared with a nonsupplemented group. Furthermore, Xing et al. (2011) obtained the same result using broiler chickens fed a diet supplemented with betaine. The fat-lowering effect of betaine was elucidated by Zhan et al. (2006) and Xing et al. (2011) who showed that betaine lowered the body fat content in broilers by increasing the activity of HSL and reducing the activities of FAS and LPL in the abdominal fat.

L-Carnitine, which transports long-chain fatty acids across the inner mitichondrial membrane during $\beta$-oxidation (the first step in fatty acid oxidation: catabolism), is a water soluble zwitterionic compound, which is synthesized in vivo from lysine and methionine (Golzar Adabi et al., 2011). Because of its role in transporting long-chain fatty acids across the inner mitichondrial membrane during $\beta$-oxidation, it appears that L-carnitine may play an important role in reducing fat deposition in modern strains of broiler chickens. Rabie and Szilagyi (1998) reported that the inclusion of L-carnitine in broiler diets at $50 \mathrm{mg} / \mathrm{kg}$ for five weeks from 18 to 53 days of age caused a significant reduction in abdominal fat deposition. Arslan et al. (2003, 2004) found that feeding Turkish native ducks and geese the same diet supplemented with 200 or $100 \mathrm{mg} \mathrm{L}$-carnitine in drinking water, respectively, led to a reduction in the abdominal fat percentage. Xu et al. (2003) showed that the abdominal fat expressed as a percentage of live weight was significantly reduced by the addition of $50 \mathrm{mg} / \mathrm{kg} \mathrm{L}-$ carnitine to the diets of broiler chickens. Feeding female broiler breeders a diet supplemented with $25 \mathrm{mg} / \mathrm{kg} \mathrm{L}$ carnitine also reduced the carcass fatness of their progeny (Kidd et al., 2005). Therefore, dietary L-carnitine supplementation decreases abdominal fat deposition in meat-type poultry by reducing the activities of G-6-PDH, MDH (lipogenic enzymes), and LPL in the abdominal fat, as suggested by Xu et al. (2003).

Probiotics is a name given to live microorganisms that have a positive effect on animal health. Santoso et al. (1995) indicated that probiotics can be added to broiler diets to reduce body fat deposition and carcass cholesterol, which suggests that probiotics beneficially regulate lipid metabolism. To test the findings of Santoso et al. (1995), Kalavathy et al. (2003) investigated the effects of Lactobacillus cultures on the abdominal fat traits and lipid profiles of broiler chickens. They found that feeding chickens aged 14 to 42 days on diets supplemented with a mixture of 12 Lactobacillus strains at $0.1 \%$ significantly reduced the serum triglyceride concentration, with a concomitant decrease in abdominal fat traits. Homma and Shinohara (2004) also reported that the inclusion of probiotic Bacillus cereus toyoi in the diets of male quails from 27 to 55 days of age for four weeks significantly reduced abdominal fat accumulation. Kalavathy et al. (2006) found that Lactobacillus cultures reduced the abdominal fat traits and also improved the carcass quality by reducing carcass fatness. Similarly, Yamamoto et al. (2007) showed that dietary supplementation with Aspergillus awamori-fermented distillery by-product, at $1.0 \%$ significantly lowered abdominal fat deposition in broiler chickens. Cao et al. (2012) found that feeding broiler chickens a diet supplemented with Aspergillus nigerfermented Ginkgo biloba leaves at $0.5 \%$ and $1.0 \%$ in the starter and grower phases, respectively, significantly reduced abdominal fat accumulation. Thus, Saleh et al. (2012) examined the effects of including Aspergillus awamori at $0.05 \%$ or $0.20 \%$ on abdominal fat deposition. They found that the inclusion of Aspergillus awamori at $0.05 \%$ was sufficient to induce a significant reduction in the abdominal fat percentage. In a comparison of the effects of Aspergillus awamori and Aspergillus niger on abdominal fat traits in chickens, Saleh et al. (2011) showed that Aspergillus awamori at $0.01 \%$ or Aspergillus niger at $0.05 \%$ in the diet significantly reduced abdominal fat deposition. Therefore, the deposition of poultry abdominal fat could be reduced by supplementing probiotics in the diet because probiotics inhibit lipid biosynthesis (Santoso et al., 1995; Yamamoto et al., 2007) and promote fatty acid catabolism (Homma and Shinohara, 2004).

Polysavone is extracted from alfalfa and contains $18.63 \%$ polysaccharides, $5.58 \%$ triterpenoid saponins, and $5.89 \%$ flavonoids. It has been implicated in the reduction of poultry abdominal fat deposition by downregulating the activity levels of ACC and FAS (Dong et al., 2007; Deng et al., 2012).

Green tea contains high levels of polyphenols, which are thought to be useful as antiobesity compounds. It has been reported that the inclusion of green tea powder at $0.5 \%$ in the diets of broiler chickens significantly reduced abdominal fat traits by inhibiting hepatic lipogenesis (Biswas and Wakit, 2001).

Chitooligosaccharide, which has a low molecular weight, low viscosity, and acceptable solubility, is an oligosaccharide that can be added to the diets of broiler chickens at $0.4 \%$ to reduce abdominal fat deposition (Zhou et al., 2009). Chitooligosaccharide lowered body fat deposition by reducing lipid uptake via the suppression of the enzyme activity of pancreatic lipase (Kang et al., 2012).

Chitosan is a linear polysaccharide with low viscosity that is produced commercially from crab and shrimp shell waste. Chitosan has been used to reduce the fat content in the abdominal area of poultry by inhibiting the absorption of dietary fat via the suppression of lipase activity due to a reduction in the levels of bile acids (Kobayashi and Itoh, 1991; Kobayashi et al., 2002).

Ginseng, the root of Panax ginseng C. A. Meyer, is a well-known traditional herbal drug in East Asian countries, which has been used to treat several heart diseases and 
metabolic diseases (Yan et al., 2011a). Ginseng has many bioactive components such as saponins that inhibit lipogenesis in chickens (Qureshi et al., 1983). The blood triglyceride concentration was significantly reduced by adding wild ginseng adventitious root to the diets of laying hens (Yan et al., 2011a). This reduced the availability of blood triglyceride for transport and deposition in the abdominal areas of poultry. Yan et al. (2011b) showed that the inclusion of wild ginseng adventitious root meal at $0.3 \%$ in the diets of broiler chickens led to a significant reduction in abdominal fat deposition.

Thyme (Thymus vulgaris) is a medicinal herb that can be used as a natural alternative to antibiotics in poultry production (Khan et al., 2012) but it also has inhibitory effects on abdominal fat traits in broiler chickens (AlKassie, 2009; Abdulkarimi et al., 2011). Al-Kassie (2009) showed that adding $200 \mathrm{ppm}$ thyme oil to the diets of broiler chickens during the fatting period (42 days) caused a significant reduction in the abdominal fat percentage compared with the control diet. Abdulkarimi et al. (2011) reported that adding $0.6 \%$ thyme extract to drinking water significantly reduced the accumulation of fat in the abdominal areas of broiler chickens. The reduction in the abdominal fat traits caused by thyme supplementation may have been attributable to the saponins in thyme (Abdulkarimi et al., 2011), which have inhibitory effects on lipogenesis (Qureshi et al., 1983).

Dihydropyridine, which has been approved as an antioxidant in poultry feed (Bakutis and Bukis, 1984; Zou et al., 2007; Niu et al., 2010; 2011), can reduce abdominal fat traits in laying hens, broiler chickens, and broiler breeder hens (Zou et al., 2007; Niu et al., 2010; 2011). Zou et al. (2007) found that feeding laying hens a diet supplemented with $300 \mathrm{mg} / \mathrm{kg}$ dihydropyridine for three months significantly reduced abdominal fat deposition by increasing the activity of HSL in the abdominal fat. Niu et al. (2010) found that adding $100 \mathrm{mg} / \mathrm{kg}$ dihydropyridine to broiler diets from one to 42 days of age was sufficient to suppress the activities of LPL in abdominal fat, as well as MDH and G-6-PDH in the liver, and to increase the levels of HSL in abdominal fat, thereby reducing the abdominal fat percentage. In broiler breeder hens, Niu et al. (2011) showed that the inclusion of dihydropyridine at $200 \mathrm{mg} / \mathrm{kg}$ in the diet was sufficient to reduce the accumulation of fat in the abdomen area by simulating the activity of HSL in the abdominal fat.

Alpha lipoic acid is a naturally occurring compound that has beneficial effects on poultry production. Experimental evidence in poultry suggests that dietary alpha lipoic acid supplementation alleviates oxidative stress, enhances the antioxidant capacity, improves meat quality, and lowers body fat deposition (Zhang et al., 2009b; Chen et al., 2011; Halici et al., 2012; El-Senousey et al., 2013). Zhang et al. (2009b) reported that diets supplemented with $900 \mathrm{mg} / \mathrm{kg}$ alpha lipoic acid from one to 42 days of age reduced the abdominal fat content in broiler chickens. A similar result was reported by El-Senousey et al. (2013) who fed broiler chickens from 21 to 42 days of age using a diet supplemented with $800 \mathrm{mg} / \mathrm{kg}$ alpha lipoic acid. The fatlowering effect of alpha lipoic acid may have been due to increased fatty acid $\beta$-oxidation (Shen et al., 2007) and/or elevated HSL activity (Fernandez-Galilea et al., 2012).

\section{Feed restriction on abdominal fat}

Limiting the feed intake in avian species using various methods (quantitative or qualitative feed restriction) has successfully addressed many problems that affect poultry farms due to intensive selection, e.g., fatness. Plavnik and Hurwitz (1985; 1991) showed that quantitative feed restriction was effective for reducing the abdominal fat content compared with full feeding. Santoso et al. (1993) also showed that quantitative feed restriction for 10 days (beginning on the first day in the second week of age for female broiler chickens) led to a significant reduction in the abdominal fat percentage and total body fat deposition. It is well known that female broiler chickens tend to deposit more fat than male broiler chickens. Moreover, Tan and Othani (2000) demonstrated that quantitative (50\% of full feed) or qualitative (diet diluted with $50 \%$ rice hulls) feed restriction in White Pekin ducks for six days starting at eight days of age caused a significant reduction in body fat deposition at the end of the fatting period compared with full feeding. Rezaei et al. (2010) and Wu et al. (2012) confirmed that qualitative feed restriction in modern broiler chickens and meat-type ducks reduced body fat deposition. Rezaei et al. (2010) found that the inclusion of rice hulls in the diet of broiler chickens at a level of $20 \%$ for five days starting at 16 days of age significantly reduced the abdominal fat and total body fat deposition. $\mathrm{Wu}$ et al. (2012) showed that meat-type ducks fed a diet diluted with $40 \%$ rice hulls for seven days starting at eight days of age led to a significant improvement in the meat quality by reducing the fat depots, including abdominal fat and skin fat. In broiler chickens, Santoso (2001) found there was a significant depression in the abdominal fat percentage and in the accumulation of fat in the body at market weight by limiting the feed intake to $25 \%$ of the ad libitum level for nine days starting on the first day of the second week of age, in a comparison of different levels of feed restriction and full feed. Similarly, Chen et al. (2012) showed that feeding chickens a diet containing $70 \%$ of their energy requirements by limiting the feed consumption to $80 \%$ of the full feed significantly reduced the abdominal fat pad and the thickness of subcutaneous fat compared with the control.

Restricted feed consumption lowered the body fat content in meat-type chickens and ducks and also reduced 
Table 1. Some additives used in poultry diets to reduce the abdominal fat content

\begin{tabular}{llll}
\hline Item & Amount (g/kg feed) & \multicolumn{1}{c}{ Mechanism ${ }^{1}$} & Source \\
\hline L-Arginine & 2.5 (Broiler chickens) & Lipogenesis $\downarrow$ & Fouad et al., 2013; Wu et al., 2011 \\
& 10.0 (Ducks) & Lipolysis $\uparrow$ & \\
L-Carnitine & 0.05 (Broiler chickens) & $\begin{array}{l}\text { Lipogenesis } \downarrow \\
\text { Lipotein lipase } \downarrow\end{array}$ & Xu et al., 2003 \\
Probiotics & 1.0 Lactobacillus strains, & Lipogenesis $\downarrow$ & Kalavathy et al., 2003; \\
& 0.1 Aspergillus awamori, & & Saleh et al., 2011;2012; \\
& or 0.5 Aspergillus niger & & Santoso et al., 1995; \\
& (Broiler chickens) & & Yamamoto et al., 2007 \\
Polysavone & 0.6 (Broiler chickens) & Lipogenesis $\downarrow$ & Dong et al., 2007; Deng et al., 2012 \\
Green tea & 5.0 (Broiler chickens) & Lipogenesis $\downarrow$ & Biswas and Wakit, 2001 \\
Chitooligosaccharide & 4.0 (Broiler chickens) & Lipase $\downarrow$ & Zhou et al., 2009; Kang et al., 2012 \\
Chitosan & 50 (Broiler chickens) & Lipase $\downarrow$ & Kobayashi and Itoh, 1991; \\
& & & Kobayashi et al., 2002 \\
Ginseng & 3.0 (Broiler chickens) & Lipogenesis $\downarrow$ & Yan et al., 2011b; Qureshi et al., 1983 \\
Thyme & 0.2 (Broiler chickens) & Lipogenesis $\downarrow$ & Al-Kassie, 2009; Qureshi et al., 1983 \\
Dihydropyridine & 0.3 (Laying hens) & Lipolysis $\uparrow$ & Zou et al., 2007; Niu et al., 2010;2011 \\
& 0.1 (Broiler chickens) & & \\
Alpha lipoic acid & 0.2 (Broiler breeder hens) & Lipogenesis $\downarrow$ & \\
& 0.8 (Broiler chickens) & Lipolysis $\uparrow$ & El-Senousey et al., 2013; Fernandez-Galilea \\
& & & et al., 2012; Shen et al., 2007
\end{tabular}

${ }^{1} \uparrow$, enhanced; $\downarrow$, inhibited.

fat accumulation in broiler breeders (Richards et al., 2003). Several studies have tested why feed restriction reduces the body fat content in poultry (Santoso et al., 1993; Tan and Othani, 2000; Yang et al., 2010; Wu et al., 2012). Santoso et al. (1993) suggested that quantitative feed restriction reduces fat deposition by inhibiting the activity of the ratelimiting enzyme during lipogenesis (ACC) in the livers of broiler chickens. Tan and Othani (2000) found that quantitative or qualitative feed restriction decreased the activities of the main lipogenic enzymes, including ACC and FAS, in the livers of White Pekin ducks. Wu et al. (2012) confirmed that feed restriction lowered the body fat content by decreasing the hepatic activity of MDH, G-6PDH, and FAS enzymes. Santoso et al. (1993), Tan and Othani (2000), and Wu et al. (2012) confirmed the study of Zhong et al. (1995) who showed that feed restriction significantly reduced hepatic lipogenesis (in an in vitro study). Yang et al. (2010) suggested that feed restriction regulates body fat deposition to promote lean meat production by reducing fatty acid biosynthesis and elevating fatty acid $\beta$-oxidation. Therefore, feed restriction reduces fat deposition by inhibiting hepatic lipogenesis and elevating fatty acid oxidation. Zhong et al. (1995) found that early feed restriction significant reduced abdominal fat deposition because of a significant decrease in the number of abdominal adipose cells. Thus, the application of quantitative or qualitative feed restriction in commercial farms could be an effective method for reducing the level of undesirable fat in modern strains of poultry.

\section{CONCLUSIONS}

First, poultry diets should be formulated as recommended by the guidelines for specific strains to avoid the problems of fatness in poultry. Second, replacing saturated fatty acids with polyunsaturated fatty acids (n-3 and n-6) or CLA, applying feed restriction, or including additives (Table 1) in poultry diets can also help to reduce abdominal fat deposition. However, the fat-reducing effects of nutritional factors have not been fully elucidated. Thus, Figure 1 and Table 1 may be helpful for appreciating our current understanding of the mechanism (s) underlying the effects of nutritional factors that beneficially regulate abdominal fat deposition and to identify the studies required to elucidate the fat-reducing effects of nutritional factors in poultry.

\section{ACKNOWLEDGMENTS}

This work was funded by Faculty of Agriculture, Cairo University, Egypt.

\section{REFERENCES}

Abdulkarimi, R., M. Daneshyar, and A. Aghazadeh. 2011. Thyme (Thymus vulgaris) extract consumption darkens liver, lowers blood cholesterol, proportional liver and abdominal fat weights in broiler chickens. Ital. J. Anim. Sci. 10:101-105.

Adams, K. A. and A. J. Davis. 2001. Dietary protein concentration regulates the mRNA expression of chicken hepatic malic enzyme. J. Nutr. 131:2269-2274. 
Al-Daraji, H. J., A. A. Al-Mashadani, W. K. Al-Hayani, A. S. AlHassani, and H. A. Mirza. 2011. Influence of in ovo injection of L-arginine on productive and physiological performance of quails. Res. Opin. Anim. Vet. Sci. 7:463-467.

Al-Kassie, G. A. M. 2009. Influence of two plant extracts derived from thyme and cinnamon on broiler performance. Pak. Vet. J. 29:169-173.

Andi, M. A. 2012. Effects of additional DL-methionine in broiler starter diet on blood lipids and abdominal fat. Afr. J. Biotechnol. 11:7579-7581.

Arslan, C., M. Citil, and M. Saatci. 2003. Effect of L-carnitine administration on growth performance, carcass traits, blood serum parameters and abdominal fatty acid composition of ducks. Arch. Anim. Nutr. 57:381-388.

Arslan, C., M. Citil, and M. Saatci. 2004. Effects of L-carnitine administration on growth performance, carcass traits, serum lipids and abdominal fatty acid compositions of geese. Rev. Med. Vet. 155:315-320.

Attia, Y. A. 2003. Performance, carcass characteristics, meat quality and plasma constituents of meat type drakes fed diets containing different levels of lysine with or without a microbial phytase. Arch. Anim. Nutr. 57:39-48.

Back, D. W., M. J. Goldman, J. E. Fisch, R. S. Ochs, and A. G. Goodridge. 1986. The fatty acid synthase gene in avian liver. Two mRNAs are expressed and regulated in parallel by feeding, primarily at the level of transcription. J. Biol. Chem. 261:4190-4197.

Badinga, L., K. T. Selberg, A. C. Dinges, C. W. Comer, and R. D. Miles. 2003. Dietary conjugated linoleic acid alters hepatic lipid content and fatty acid composition in broiler chickens. Poult. Sci. 82:111-116.

Baéza, E. and E. Le Bihan-Duval. 2013. Chicken lines divergent for low or high abdominal fat deposition: A relevant model to study the regulation of energy metabolism. Animal 7:965-973.

Baião, N. C. and L. J. C. Lara. 2005. Oil and fat in broiler nutrition. Brazilian J. Poult. Sci. 7:129-141.

Bakutis, B. and Y. Bukis. 1984. Antioxidants in feeding of broiler ducks. J. Ptitsevodstvo10:21-22.

Becker, W. A., J. V. Spencer, L. W. Mirosh, and J. A. Verstrate. 1979. Prediction of fat and fat free live weight in broiler chickens using back skin fat, abdominal fat and live body weight. Poult. Sci. 58:835-842.

Berri, C., J. Besnard, and C. Relandeau. 2008. Increasing dietary lysine increases final $\mathrm{pH}$ and decreases drip loss of broiler breast meat. Poult. Sci. 87:480-484.

Biswas, Md. A. H. and M. Wakita. 2001. Effect of dietary Japanese green tea powder supplementation on feed utilization and carcass profiles in broilers. J. Poult. Sci. 38:50-57.

Butterwith, S. C. 1989. Contribution of lipoprotein lipase activity to the differential growth of three adipose tissue depots in young broiler chickens. Br. Poult. Sci. 30:927-933.

Cao, F. L., X. H. Zhang, W. W. Yu, L. G. Zhao, and T. Wang. 2012. Effect of feeding fermented Ginkgo biloba leaves on growth performance, meat quality, and lipid metabolism in broilers. Poult. Sci. 91:1210-1221.

Chen, P., Q. G. Ma, C. Ji, J.Y. Zhang, L. H. Zhao, Y. Zhang, and Y. Z. Jie. 2011. Dietary lipoic acid influences antioxidant capability and oxidative status of broilers. Int. J. Mol. Sci.
12:8476-8488.

Chen, W., Y. M. Guo, Y. Q. Huang, Y. H. Shi, C. X. Zhang, and J. W. Wang. 2012. Effect of energy restriction on growth, slaughter performance, serum biochemical parameters and Lpin2/WDTC1 mRNA expression of broilers in the later phase. J. Poult. Sci. 49:12-19.

Choct, M., A. Naylor, O. Hutton, and J. Nolan. 2000. Increasing efficiency of lean tissue composition in broiler chickens. A Report for the Rural Industries Research and Development Corporation. Publication No 98/123. https://rirdc.infoservices. com.au/downloads/98-123. Accessed September 20, 2013.

Choi, J., J. Song, Y. M. Choi, D. J. Jang, E. Kim, I. Kim, and K. M. Chee. 2006. Daidzein modulations of apolipoprotein B and fatty acid synthase mRNA expression in chick liver vary depending on dietary protein levels. Asian Australas. J. Anim. Sci. 19:236-244.

Collin, A., R. D. Malheiros, V. M. B. Moraes, P. Van As, V. M. Darras, M. Taouis, E. Decuypere, and J. Buyse. 2003. Effects of dietary macronutrient content on energy metabolism and uncoupling protein mRNA expression in broiler chickens. Br. J. Nutr. 90:261-269.

Corzo, A., E. T. Jr. Moran, and D. Hoehler. 2003. Arginine need of heavy broiler males: Applying the ideal protein concept. Poult. Sci. 82:402-407.

Corzo, A., M. T. Kidd, W. A. Dozier, L. A. Shack, and S. C. Burgess. 2006. Protein expression of pectoralis major muscle in chickens in response to dietary methionine status. Br. J. Nutr. 95:703-708.

Crespo, N. and E. Esteve-Garcia. 2001. Dietary fatty acid profile modifies abdominal fat deposition in broiler chickens. Poult. Sci. 80:71-78.

Crespo, N. and E. Esteve-García. 2002a. Dietary polyunsaturated fatty acids decrease fat deposition in separable fat depots but not in the remainder carcass. Poult. Sci. 81:512-518.

Crespo, N. and E. Esteve-García. 2002b. Dietary linseed oil produces lower abdominal fat deposition but higher de novo fatty acid synthesis in broiler chickens. Poult. Sci. 81:15551562.

Cui, H. X., M. Q. Zheng, R. R. Liu, G. P. Zhao, J. L. Chen, and J. Wen. 2012. Liver dominant expression of fatty acid synthase (FAS) gene in two chicken breeds during intramuscular-fat development. Mol. Biol. Rep. 39:3479-3484.

Deng, W., X. F. Dong, J. M. Tong, T. H. Xie, and Q. Zhang. 2012. Effects of an aqueous alfalfa extract on production performance, egg quality and lipid metabolism of laying hens. J. Anim. Physiol. Anim. Nutr. 96:85-94.

Dong, X. F., W. W. Gao, J. M. Tong, H. Q. Jia, R. N. Sa, and Q. Zhang. 2007. Effect of polysavone (alfalfa extract) on abdominal fat deposition and immunity in broiler chickens. Poult. Sci. 86:1955-1959.

Du, M. and D. U. Ahn. 2002. Effect of dietary conjugated linoleic acid on the growth rate of live birds and on the abdominal fat content and quality of broiler meat. Poult. Sci. 81:428-433.

Eaton, S. 2002. Control of mitochondrial beta-oxidation flux. Prog. Lipid. Res. 41:197-239.

El-Senousey, H. K., A. M. Fouad, J. H. Yao, Z. G. Zhang, and Q. W. Shen. 2013. Dietary alpha lipoic acid improves body composition, meat quality and decreases collagen content in 
muscle of broiler chickens. Asian Australas. J. Anim. Sci. 26:394-400.

Emmerson, D. A. 1997. Commercial approaches to genetic selection for growth and feed conversion in domestic poultry. Poult. Sci. 76:1121-1125.

Fan, H. P., M. Xie, W. W. Wang, S. S. Hou, and W. Huang. 2008. Effects of dietary energy on growth performance and carcass quality of white growing pekin ducks from two to six weeks of age. Poult. Sci. 87:1162-1164.

Fernandez-Galilea, M., P. Perez-Matute, P. L. Prieto-Hontoria, J. A. Martinez, and M. J. Moreno-Aliaga. 2012. Effects of lipoic acid on lipolysis in 3T3-L1 adipocytes. J. Lipid Res. 53:22962306.

Ferrini, G., E. G.Manzanilla, D. Menoyo, E. Esteve-Garcia, M. D. Baucells, and A. C. Barroeta. 2010. Effects of dietary n-3 fatty acids in fat metabolism and thyroid hormone levels when compared to dietary saturated fatty acids in chickens. Livest. Sci. 131:287-291.

Flock, D. K., K. F. Laughlin, and J. Bentley. 2005. Minimizing losses in poultry breeding and production: How breeding companies contribute to poultry welfare. World's Poult. Sci. J. 61:227-237.

Fouad, A. M., H. K. El-Senousey, X. J. Yang, and J. H. Yao. 2012. Role of dietary L-arginine in poultry production. Int. J. Poult. Sci. 11:718-729.

Fouad, A. M., H. K. El-Senousey, X. J. Yang, and J. H. Yao. 2013. Dietary L-arginine supplementation reduces abdominal fat content by modulating lipid metabolism in broiler chickens. Animal 7:1239-1245.

Golzar Adabi, S. H., R. G. Cooper, N. Ceylan, and M. Corduk. 2011. L-carnitine and its functional effects in poultry nutrition. World's Poult. Sci. J. 67:277-296.

Grisoni, M. L., G. Uzu, M. Larbier, and P. A. Geraert. 1991. Effect of dietary lysine on lipogenesis in broilers. Reprod. Nutr. Dev. 31:683-690.

Halici, M., H. Imik, M. Koç, and R. Gümüş. 2012. Effects of $\alpha-$ lipoic acid, vitamins $\mathrm{E}$ and $\mathrm{C}$ upon the heat stress in Japanese quails. J. Anim. Physiol. Anim. Nutr. 96:408-415.

Havenstein, G. B., P. R. Ferket, and M. A. Qureshi. 2003. Growth, livability and feed conversion of 1957 versus 2001 broilers when fed representative 1957 and 2001 broiler diets. Poult. Sci. 92:1500-1508.

Hermier, D. 1997. Lipoprotein metabolism and fattening in poultry. J. Nutr. 127:805-808

Homma, H. and T. Shinohara. 2004. Effects of probiotic Bacillus cereus toyoi on abdominal fat accumulation in the Japanese quail (Coturnix japonica). Anim. Sci. J. 75:37-41.

Jlali, M., V. Gigaud, S. Métayer-Coustard, N. Sellier, S. Tesseraud, E. Le Bihan-Duval, and C. Berri. 2012. Modulation of glycogen and breast meat processing ability by nutrition in chickens: Effect of crude protein level in 2 chicken genotypes. J. Anim. Sci. 90:447-455.

Kalavathy, R., N. Abdullah, S. Jalaludin, and Y. W. Ho. 2003. Effects of Lactobacillus cultures on growth performance, abdominal fat deposition, serum lipids and weight of organs of broiler chickens. Br. Poult. Sci. 44:139-144.

Kalavathy, R., N. Abdullah, S. Jalaludin, M. C. Wong, and Y. W. Ho. 2006. Effects of Lactobacillus feed supplementation on cholesterol, fat content and fatty acid composition of the liver, muscle, and carcass of broiler chickens. Anim. Res. 55:77-82.

Kang, N. H., W. K. Lee, B. R. Yi, M. A. Park, H. R. Lee, S. K. Park, K. A. Hwang, H. K. Park, and K. C. Choi. 2012. Modulation of lipid metabolism by mixtures of protamine and chitooligosaccharide through pancreatic lipase inhibitory activity in a rat model. Lab. Anim. Res. 28:31-38.

Kassim, H. and S. Suwanpradit. 1996a. The effect of energy levels on the carcass composition of the broilers. Asian J. Anim. Sci. 9:331-335.

Kassim, H. and S. Suwanpradit. 1996b. The effects of dietary protein levels on the carcass composition of starter and grower broilers. Asian Australas. J. Anim. Sci. 9:261-266.

Khan, R. U., S. Naz, Z. Nikousefat, V. Tufarelli, and V. Laudadio. 2012. Thymus vulgari: alternative to antibiotics in poultry feed. World's Poult. Sci. J. 68:401-408.

Kidd, M. T., C. D. McDaniel, E. D. Peebles, S. J. Barber, A. Corzo, S. L. Branton, and J. C. Woodworth. 2005. Breeder hen dietary L-carnitine affects progeny carcase traits. Br. Poult. Sci. 46:97103.

Klimis-Taventzis, D. J., P. M. Kris-Etherton, and R. M. Jr. Leach. 1983. The effect of dietary manganese deficiency on cholesterol and lipid metabolism in the estrogen-treated chicken and the laying hen. J. Nutr. 113:320-327.

Kobayashi, S. and H. Itoh. 1991. Effect of dietary chitin and chitosan on growth and abdominal fat deposition in chicks. J. Poult. Sci. 28:88-94.

Kobayashi, S., Y. Terashima, and H. Itoh. 2002. Effect of dietary chitosan on fat deposition and lipase activity in digesta in broiler chickens. Br. Poult. Sci. 43:270-273.

Li, S., L. Lin, H. Shoufeng, W. Yanping, Z. Liyang, L. Songbai, L. Bin, L. Kui, and X. Luo. 2011. Dietary manganese modulates expression of the manganese-containing superoxide dismutase gene in chickens. J. Nutr. 141:189-194.

Lu, L., C. Ji, X. G. Luo, B. Liu, and S. X. Yu. 2006. The effect of supplemental manganese in broiler diets on abdominal fat deposition and meat quality. Anim. Feed Sci. Technol. 129:4959.

Lu, L., X. G. Luo, C. Ji, B. Liu, and S. X. Yu. 2007. Effect of manganese supplementation and source on carcass traits, meat quality, and lipid oxidation in broilers. J. Anim. Sci. 85:812822

Moran, E. T. and S. F. Bilgili. 1990. Processing losses, carcass quality and meat yields of broiler chickens receiving diets marginally deficient to adequate in lysine prior to marketing. Poult. Sci. 69:702-710.

Nasr, J. and F. Kheiri. 2011. Effect of different lysine levels on Arian broiler performances. Ital. J. Anim. Sci. 10:170-174.

Newman, R. E., W. L. Bryden, E. Fleck, J. R. Ashes, W. A. Buttemer, L. H. Storlien, and J. A. Downing. 2002. Dietary n-3 and n- 6 fatty acids alter avian metabolism: metabolism and abdominal fat deposition. Br. J. Nutr. 88:11-18.

Niu, Z. Y., F. Z. Liu, Y. N. Min, and W. C. Li. 2010. Effects of dietary dihydropyridine supplementation on growth performance and lipid metabolism of broiler chickens. Czech J. Anim. Sci. 55:116-122.

Niu, Z. Y., Y. N. Min, H. Y. Wang, J. Zhang, W. C. Li, L. Li, and F. Z. Liu. 2011. Effects of dietary dihydropyridine on laying 
performance and lipid metabolism of broiler breeder hens. S. Afr. J. Anim. Sci. 41:331-336.

NRC (National Research Council). 1994. Nutrient Requirements for Poultry. 9th Edn. National Academy Press, Washington DC, USA.

Plavnik, I. and S. Hurwitz. 1985. The performance of broiler chicks during and following a severe feed restriction at an early age. Poult. Sci. 64:348-355.

Plavnik, I. and S. Hurwitz. 1991. Response of broiler chickens and turkey poults to food restriction of varied severity during early life. Br. Poult. Sci. 32:343-352.

Qureshi, A. A., Z. Z. Din, N. Abuirmeleh, W. C. Burger, Y. Ahmad, and C. E. Elson. 1983. Suppression of cholesterogenesis and reduction of LDL cholesterol by dietary ginseng and its fractions in chicken liver. Atherosclerosis 48:81-94.

Rabie, M. H. and M. Szilagyi. 1998. Effects of L-carnitine supplementation of diets differing in energy levels on performance, abdominal fat content, and yield and composition of edible meat of broilers. Br. J. Nutr. 80:391-400.

Rezaei, M. and H. Hajati. 2010. Effect of diet dilution at early age on performance, carcass characteristics and blood parameters of broiler chicks. Ital. J. Anim. Sci. 9:93-100.

Richards, M. P., S. M. Poch, C. N. Coon, R. W. Rosebrough, C. M. Ashwellm, and J. P. McMurtry. 2003. Feed restriction significantly alters lipogenic gene expression in broiler breeder chickens. J. Nutr. 133:707-715.

Rosebrough, R. W., B. A. Russell, and M. P. Richards. 2008. Short term changes in expression of lipogenic genes in broilers (Gallus gallus). Comp. Biochem. Physiol. Part A Mol. Integr. Physiol. 149:389-395.

Rosebrough, R. W., B. A. Russell, and M. P. Richards. 2011. Further studies on short-term adaptations in the expression of lipogenic genes in broilers. Comp. Biochem. Physiol. Part A Mol. Integr. Physiol. 159:1-6.

Rosebrough, R. W., S. M. Poch, B. A. Russell, and M. P. Richards. 2002. Dietary protein regulates in vitro lipogenesis and lipogenic gene expression in broilers. Comp. Biochem. Physiol. Part A Mol. Integr. Physiol. 132:423-431.

Royan, M., G. Y. Meng, F. Othman, A. Q. Sazili, and B. Navidshad. 2011. Effects of conjugated linoleic acid, fish oil and soybean oil on PPARs $(\alpha \& \gamma)$ mRNA expression in broiler chickens and their relation to body fat deposits. Int. J. Mol. Sci. 12: 8581-8595.

Saleh, A. A., Y. Z. Eid, T. A. Ebeid, A. Ohtsuka, K. Hioki, M. Yamamoto, and K. Hayashi. 2012. The modification of the muscle fatty acid profile by dietary supplementation with Aspergillus awamori in broiler chickens. Br. J. Nutr. 108:15961602.

Saleh, A. A., Z. Eid, and K. Hayashi. 2011. Effects of feeding Aspergillus awamori and Aspergillus niger on growth performance and meat quality in broiler chickens. J. Poult. Sci. 48: 201-206.

Sands, J. S. and M. O. Smith. 1999. Broilers in heat stress conditions: Effects of dietary manganese proteinate or chromium picolinate supplementation. J. Appl. Poult. Res. 8: 280-287.

Santoso, U. 2001. Effects of early feed restriction on growth, fat accumulation and meat composition in unsexed broiler chickens. Asian Australas. J. Anim. Sci. 14:1585-1591.
Santoso, U., K. Tanaka, and S. Ohtania. 1995. Effect of dried Bacillus subtilis culture on growth, body composition and hepatic lipogenic enzyme activity in female broiler chicks. Br. J. Nutr. 74:523-529.

Santoso, U., K. Tanaka, S. Ohtani, and B. S. Youn. 1993. Effects of early feed restriction on growth performance and body composition in broilers. Asian Australas. J. Anim. Sci. 6:401410.

Sanz, M., A. Flores, and C. J. Lopez-Bote. 2000a. The metabolic use of energy from dietary fat in broilers is affected by fatty acid saturation. Br. Poult. Sci. 41:61-68.

Sanz, M., A. Flores, P. Perez de Ayala, and C. J. Lopez-Bote. 1999. Higher lipid accumulation in broilers fed on saturated fats than in those fed on unsaturated fats. Br. Poult. Sci. 40:95-101.

Sanz, M., C. J. Lopez-Bote, D. Menoyo, and J. M. Bautista. 2000b. Abdominal fat deposition and fatty acid synthesis are lower and $\beta$-oxidation is higher in broiler chickens fed diets containing unsaturated rather than saturated fat. J. Nutr. 130: 3034-3037.

Sato, K., H. Abe, T. Kono, M. Yamazaki, K. Nakashima, T. Kamada, and Y. Akiba. 2009. Changes in peroxisome proliferator-activated receptor gamma gene expression of chicken abdominal adipose tissue with different age, sex and genotype. Anim. Sci. J. 80:322-327.

Shen, Q. W., M. J. Zhu, J. Tong, J. Ren, and M. Du. 2007. $\mathrm{Ca}^{2+} /$ calmodulin-dependent protein kinase kinase is involved in AMP-activated protein kinase activation by alpha-lipoic acid in $\mathrm{C}_{2} \mathrm{C}_{12}$ myotubes. Am. J. Physiol. Cell Physiol. 293: C1395-C1403.

Simon, O., K. Manner, K. Schafer, A. Sagredos, and K. Eder. 2000. Effect of conjugated linoleic acid on protein-to-fed proportion, fatty acids and plasma lipids in broilers. Eur. J. Lipid Sci. Technol. 102:402-410.

Simopoulos, A. P. 2000. Human requirement for N-3 polyunsaturated fatty acids. Poult. Sci. 79:961- 970.

Su, S. Y., M. V. Dodson, X. B. Li, Q. F. Li, H. W. Wang, and Z. Xie. 2009. The effects of dietary betaine supplementation on fatty liver performance, serum parameters, histological changes, methylation status and the mRNA expression level of Spot $14 \alpha$ in Landes goose fatty liver. Comp. Biochem. Physiol. Part A Mol. Integr. Physiol. 154:308-314.

Szymczyk, B., P. M. Pisulewski, W. Szczurek, and P. Hanczakowski. 2001. Effects of conjugated linoleic acid on growth performance, feed conversion efficiency, and subsequent carcass quality in broiler chickens. Br. J. Nutr. 85:465-473.

Takahashi, K. and Y. Akiba. 1995. Effect of methionine supplementation on lipogenesis and lipolysis in broiler chickens. J. Poult. Sci. 32:99-106.

Tan, B. J. and S. Ohtani. 2000. Effect of different early feed restriction regimens on performance, carcass composition and lipid metabolism in male ducks. Anim. Sci. J. 71:586-593.

Tanaka, K., S. Ohyani, and K. Shigeno. 1983. Effect of increasing dietary energy on hepatic lipogenesis in growing chicks. II. Increasing energy by fat or protein supplementation. Poult. Sci. 62:452-458.

Tesseraud, S., I. Bouvarel, A. Collin, E. Audouin, S.Crochet, I. Seiliez, and C. Leterrier. 2009. Daily variations in dietary 
lysine content alter the expression of genes related to proteolysis in chicken pectoralis major muscle. J. Nutr. 139: 38-43.

Thomas, V. G., S. K. Mainguy, and J. P. Prevett. 1983. Predicting fat-content of geese from abdominal fat weight. J. Wildl. Manage. 47:1115-1119.

Virtanen, E. 1995. Piecing together the betaine puzzle. Feed Mix 3:12-17.

Wang, S. Z., X. X. Hu, Z. P. Wang, X. C. Li, Q. G. Wang, Y. X. Wang, Z. Q. Tang, and H. Li. 2012. Quantitative trait loci associated with body weight and abdominal fat traits on chicken chromosomes 3, 5 and 7. Genet. Mol. Res. 11:956-965.

Wang, Y. Z., Z. R. Xu, and J. Feng. 2004. The effect of betaine and DL-methionine on growth performance and carcass characteristics in meat ducks. Anim. Feed Sci. Technol. 116: 151-159.

Wang, Y., Y. Mu, H. Li, N. Ding, Q. Wang, Y. Wang, S. Wang, and N. Wang. 2008. Peroxisome proliferator-activated receptor- $\gamma$ gene: A key regulator of adipocyte differentiation in chickens. Poult. Sci. 87:226-232.

Wang, Z. Y., S. R. Shi, Q. Y. Zhou, H. M. Yang, J. M. Zou, K. N. Zhang, and H. M. Han. 2010. Response of growing goslings to dietary methionine from 28 to 70 days of age. Br. Poult. Sci. 51:118-121.

Wu, L., X. Guo, and Y. Fang. 2012. Effect of diet dilution ratio at early age on growth performance, carcass characteristics and hepatic lipogenesis of Pekin ducks. Braz. J. Poult. Sci. 14:4349.

Wu, L. Y., Y. J. Fang, and X. Y. Guo. 2011. Dietary Larginine supplementation beneficially regulates body fat deposition of meat-type ducks. Br. Poult. Sci. 52:221-226.

Xie, M., J. N. Zhao, S. S. Hou, and W. Huang. 2010. The apparent metabolizable energy requirement of White Pekin ducklings from hatch to 3 weeks of age. Anim. Feed Sci. Technol. 157: 95-98.

Xie, M., S. S. Hou, and W. Huang. 2006. Methionine requirements of male white Peking ducks from twenty-one to forty nine days of age. Poult. Sci. 85:743-746.

Xing, J., L. Kang, and Y. Jiang. 2011. Effect of dietary betaine supplementation on lipogenesis gene expression and $\mathrm{CpG}$ methylation of lipoprotein lipase gene in broilers. Mol. Biol. Rep. 38:1975-1981.

Xing, J., L. Kang, Y. Hu, Q. Xu, N. Zhang, and Y. Jiang. 2009. Effect of dietary betaine supplementation on mRNA expression and promoter $\mathrm{CpG}$ methylation of lipoprotein lipase gene in laying hens. J. Poult. Sci. 46:224-228.

Xiong, M., S. Li, Peng, Y. Feng, G. Yu, Q. Xin, and Y. Gong. 2010. Adipogenesis in ducks interfered by small interfering ribonucleic acids of peroxisome proliferator-activated receptor $\gamma$ gene. Poult. Sci. 89:88-95.

Xu, Z. R., M. Q. Wang, H. X. Mao, X. A. Zhan, and C. H. Hu. 2003. Effects of L-carnitine on growth performance, carcass composition, and metabolism of lipids in male broilers. Poult. Sci. 82:408-413.

Yalçin, S., H. Özkul, S. Özkan, R. Gous, I. Yaşa, and E. Babacanoğlu. 2010. Effect of dietary protein regime on meat quality traits and carcase nutrient content of broilers from two commercial genotypes. Br. Poult. Sci. 51:621-628.
Yamamoto, M., F. Saleh, M. Tahir, A. Ohtsuka, and K. Hayashi. 2007. The effect of Koji-fed (fermented distillery byproduct) on the growth performance and nutrient metabolizability in broiler. J. Poult. Sci. 44:291-296

Yan, L., Q. W. Meng, J. H. Lee, J. P. Wang, and I. H. Kim. 2011a. Effect of dietary wildginseng adventitious root meal on growth performance, blood profiles, relative organ weight and meat quality in broiler chickens. Asian Australas. J. Anim. Sci. 24: 258-263.

Yan, L., Q. W. Meng, X. Ao, J. P. Wang, H. D. Jang, and I. H. Kim. 2011b. Evaluation of dietary wild-ginseng adventitious root meal on egg production, egg quality, hematological profiles and egg yolk fatty acid composition in laying hens. Livest. Sci. 140:201-205.

Yang, X., J. Zhuang, K. Rao, X. Li, and R. Zhao. 2010. Effect of early feed restriction on hepatic lipid metabolism and expression of lipogenic genes in broiler chickens. Res. Vet. Sci. 89:438-444.

Yao, J. H., S. Q. Li, L. L. Zhong, S. X. Huang, W. J. Zhang, and H. B. Xi. 2006. The relative effectiveness of liquid methionine hydroxy analogue compared to DL-methionine in broilers. Asian Australas. J. Anim. Sci. 19:1026-1032.

Zhan, X. A., J. X. Li, Z. R. Xu, and R. Q. Zhao. 2006. Effects of methionine and betaine supplementation on growth performance, carcase composition and metabolism of lipids in male broilers. Br. Poult. Sci. 47:576-580.

Zhang, G. M., J. Wen, J. L. Chen, G. P. Zhao, M. Q. Zheng, and W. J. Li. 2007. Effect of conjugated linoleic acid on growth performances, carcass composition, plasma lipoprotein lipase activity and meat traits of chicken. Br. Poult. Sci. 48:217-223.

Zhang, X., B. Wang, F. Long, L. Wang, and Z. Yang. 2009a. The effect of dietary conjugated linoleic acid (CLA) on fatty acid composition and key enzymes of fatty acids oxidation in liver and muscle of geese. Turk. J. Vet. Anim. Sci. 33:215-222.

Zhang, Y., K. Hongtrakul, Q. G. Ma, L. T. Liu, and X. X. Hu. 2009b. Effects of dietary alpha-lipoic acid on anti-oxidative ability and meat quality in Arbor Acres broilers. Asian Australas. J. Anim. Sci. 22:1195-1201.

Zhong, C., H. S. Nakaue, C. Y. Hu, and L. W. Mirosh. 1995. Effect of full food and early food restriction on broiler performance, abdominal fat level, cellularity and fat metabolism in broiler chickens. Poult. Sci. 74:1636-1643.

Zhou, H., N. Deeb, C. M. Evock-Clover, C. M. Ashwel, and S. J. Lamont. 2006. Genome-wide linkage analysis to identify chromosomal regions affecting phenotypic traits in the chicken. II. Body composition. Poult. Sci. 85:1712-1721.

Zhou, J. 2008. Effect of dietary conjugated linoleic acid (CLA) on abdominal fat deposition in yellow-feather broiler chickens and its possible mechanism. Asian Australas. J. Anim. Sci. 21:1760-1765.

Zhou, T. X., Y. J. Chen, J. S. Yoo, Y. Huang, J. H. Lee, H. D. Jang, S. O. Shin, H. J. Kim, J. H. Cho, and I. H. Kim. 2009. Effects of chitooligosaccharide supplementation on performance, blood characteristics, relative organ weight, and meat quality in broiler chickens. Poult. Sci. 88:593-600.

Zou, X. T., Z. R. Xu, J. L. Zhu, X. J. Fang, and J. F. Jiang. 2007. Effects of dietary dihydropyridine supplementation on laying performance and fat metabolism of laying hens. Asian Australas. J. Anim. Sci. 20:1606-1611. 